\title{
Novel properties and applications in magnetic fluids
}

\author{
H.E. Horng ${ }^{\mathrm{a}, *}$, Chin-Yih Hong ${ }^{\mathrm{b}}$, S.Y. Yang ${ }^{\mathrm{a}}$, H.C. Yang ${ }^{\mathrm{c}}$ \\ ${ }^{a}$ Department of Physics, National Taiwan Normal University, Taipei 116, Taiwan \\ ${ }^{\mathrm{b}}$ Department of Mechanical Engineering, Da-Yeh University, Chang-Hwa 515, Taiwan \\ ${ }^{\mathrm{c}}$ Department of Physics, National Taiwan University, Taipei 106, Taiwan
}

Received 28 December 2000; accepted 3 January 2001

\begin{abstract}
In this review, we will report a series of investigations on the homemade homogeneous magnetic fluids. The most spectacular property discussed in our research is the ordered structure of the magnetic columns formed in the magnetic fluid thin film under the influence of the external magnetic field, either perpendicular or parallel to the film surface. It is worth noting that the ordered structure can be manipulated by changing the control parameters. This reveals the variability of the ordered structure. With the ordered structures, some significant magneto-optical characteristics such as magnetochromatics, birefringence, and fielddependent transmittance are generated. These optical properties form the groundwork for further development of related optical devices by using the homogeneous magnetic fluid films. (C) 2001 Elsevier Science Ltd. All rights reserved.
\end{abstract}

\section{Introduction}

In the early part of the 20th century, almost all the efforts of scientists were put into dealing with the diverse phenomena of the solid matter. The conceptual paradigm of the solid matter is the picture of a dense periodic lattice and basis of atoms. This paradigm has been spectacularly successful in describing the properties of solid matter and continued to underlie much of the ongoing work. However, in the 1960s, a particular class of materials appeared [1-7], named soft material, whose properties and behaviors are absolutely different to those of solid matter. This leads to a remarkable evolution from the solid state science to the condensed matter science. In general, the soft materials, which are characterized by the fact that they are easily deformed by external stresses, electromagnetic or gravitational fields, etc., may include fluids, liquid crystals, polymers, emulsions and colloids. With the high sensitivity and actuation, the application of soft materials is growing and showing the versatile industrial interest of these smart materials containing soft building blocks.

In the meantime, the other significant progress in science

\footnotetext{
* Corresponding author. Tel.: +886-2-8931-6233; fax: +886-22930-9439.

E-mail address: phyfv001@scc.ntnu.edu.tw (H.E. Horng).
}

and technology during this period was the sprouting of the nanoscale science and technology [8-10]. Nanoscience and technology emphasize materials of small dimensions, typically $1-100 \mathrm{~nm}$, which connect the macroscopic and the microscopic areas of research. Strict control of the chemical and structural perfection of the samples is the essence of the nanoscale technology. These novel techniques capable of fabricating nanoscale building blocks have opened up a revolutionary method of exploring material properties and device characteristics, and the work is expanding rapidly worldwide. Therefore, nanoscience is a very broad and interdisciplinary area of research and hence brings tremendous common interests of research to chemists, physicists, metallurgists and engineers. Generally, the worldwide research of nanoscience is carried out in nanoparticle, nanostructured materials and nanodevices [11]. Among these categories, the work on the dispersion of nanoparticles in liquids or polymers leads to a hybrid of nanoscience and the soft material. These materials possess properties of both soft materials and nanoparticles, and also, show fruitful commercial impacts.

Magnetic fluid (also called ferrofluid), which consists of colloidal magnetic nanoparticles dispersed with the aid of surfactants in a continuous carrier phase, is a typical hybrid of soft material and the nanoparticle. The ferrofluid was first synthesized successfully in the 1960s [12,13]. A magnetic 
powder was reduced to colloidal nanoparticles by using ballmilling grinding in the presence of a surfactant and liquid carrier. The average diameter of the dispersed particles ranges from 5 to $10 \mathrm{~nm}$. Each particle in the fluid behaves as a constant magnetic dipole moment proportional to its size and can align with an external magnetic field freely, but due to the low production efficiency through the ballmilling grinding process, another method of chemical precipitation was then developed in the 1970s [14]. Because of the improvement in the manufacture process, the ferrofluid becomes popular and hence the interest of scientists is attracted to the area of the research. This research is mainly focused on the structural forming systems of ferrofluids under magnetic fields [15-20], the magnetically induced optical properties [21-24], and the applications in mechanical related work [25-29], optical devices [30-32] or even in medicine [33].

As mentioned above, the ferrofluids contain enormous magnetic nanoparticles in the fluid, and hence can be influenced by either parallel or perpendicular magnetic field. Under parallel magnetic fields, magnetic chains were found in ferrofluid film $[24,34,35]$. This leads to the magnetic-field induced birefringence of the ferrofluid $[21,24]$, and also, the birefringence can be controlled by varying the field strength $[24,36,37]$. A similar phenomenon has been observed for the liquid crystal, which is also one of the soft materials exhibiting birefringence under or without electric field. As we know, the electric-field modulated birefringence of the liquid crystals is applied widely in optical devices, such as liquid crystal displays, switches, etc.. This suggests that there is a good chance of developing optical devices by utilizing the magnetic-field induced birefringence of ferrofluid.

On the other hand, under perpendicular fields, many researchers indicated that disordered two-dimensional lattices of magnetic columns, which are agglomerated by magnetic nanoparticles, are formed [17,38,39]. Other investigators have generated more highly ordered two-dimensional lattices in thin films of magnetic emulsions [40], ferrofluids containing non-magnetic spheres [41] or concentrated ferroluids based on a mineral solvent [42]. However, these lattices tend to solidify and therefore are not suitable for applications requiring rapid interconversion between crystalline and amorphous states. After the success in synthesizing highly homogeneous ferrofluids in our recent work [43], we found an ordered hexagonal structure pattern of magnetic columns all over the ferrofluid film under uniform perpendicular magnetic fields exceeding a critical value [19]. Later, an unprecedented structure evolution in the ferrofluid film under perpendicular fields was discovered [44]: agglomeration, disordered columns phase, multi-level ordered hexagonal structure through a transition phase. The ordered hexagonal structure of magnetic columns in the ferrofluid film results in a novel optical property of magnetochromatics $[23,45]$, which reveals that the hexa- gonal structure of magnetic columns acts as a twodimensional grating.

Owing to the versatility of the structural patterns and the magnetically induced optical properties of the ferrofluid films under external magnetic fields, the research on ferrofluid is spreading rapidly all over the world. In this report, we will first give an overview of the structural pattern formation of the magnetic columns in the ferrofluid thin film under magnetic fields, and then exhibit the framework of the application of the ferrofluid based on the remarkable optical properties caused by these magnetically induced structures.

\section{Structure in the magnetic fluid films under external magnetic fields}

To observe the structural patterns of magnetic fluids (or ferrofluids) under external magnetic fluid fields, magnetic fluid is usually sealed in a glass cell with a thickness of several to tens of micrometers. The applied magnetic field is either parallel (parallel field) or perpendicular (perpendicular field) to the plane of the film. The images of the structures in the magnetic fluid film are taken with an optical microscope and are recorded with a CCD video camera.

A variety of different pattern-forming systems of magnetic fluid films under external magnetic fields have been studied. In this section, we illustrate structure evolution in the film and the fingering behavior of magnetic microdrops under external magnetic fields.

\subsection{Structure evolution}

In this part, the structure evolution in the magnetic fluid film under parallel or perpendicular magnetic fields is separately discussed.

\subsubsection{Parallel magnetic fields}

When a magnetic fluid film is subjected to a parallel magnetic field, a portion of the magnetic particles in the fluid agglomerate to form needle-like chains in the magnetic fluid film [34,46,47]. A typical picture of the needle-like chains is shown in Fig. 1. Some researchers suggest that the agglomeration of magnetic particles is due to phase separation, which results from the thermodynamical instability of the dispersion of magnetic particles $[48,49]$. By looking upon the magnetic fluid as a binary solution of solvent molecules and colloidal particles, Taketomi et al. used Debye's critical opalescence theory to establish a thermodynamical instability theory of colloidal particles dispersion for interpreting the agglomerating behavior [16].

As the field strength is further increased, more particles participate in the agglomeration and needle-like chains are distributed randomly in the film. Yusuf et al. showed that two separated chains merge and that the chains become longer under a higher field [35]. It was also found that the 


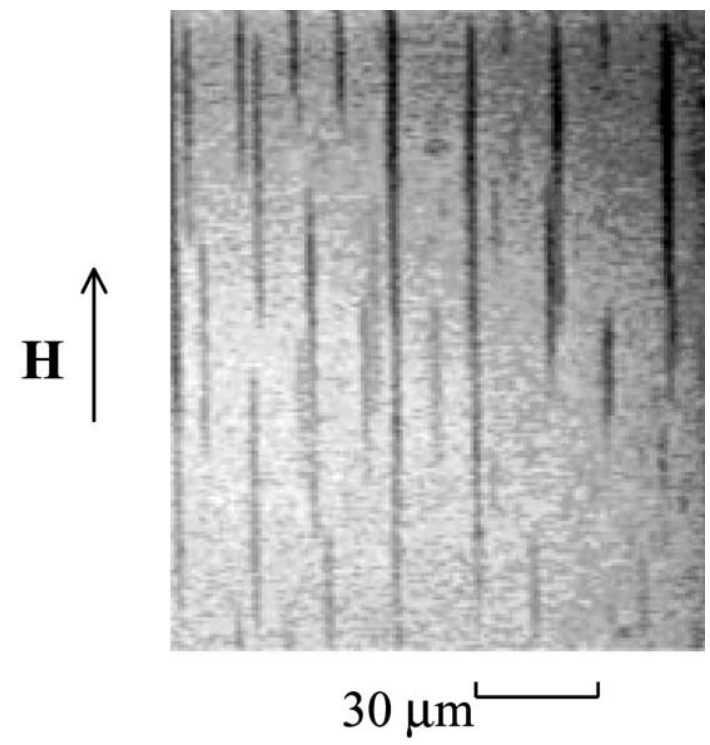

Fig. 1. Needle-like chains in the magnetic fluid film under a parallel magnetic field [16].

chains elongate when the fluid concentration is raised. With the recent success in synthesizing highly homogeneous magnetic fluid [31], a nearly one-dimensional periodic long-chain structure was obtained for magnetic fluid film under parallel magnetic fields [24,37,50,51], as shown in Fig. 2. Furthermore, the variation of the one-dimensional periodic long-chain structures under various magnetic fields was also investigated. It was observed that the spacing $s$ between two neighboring long chains and the averaged chain width $w$ decreased as the $H$ was increased. The desired final field strength $H$ was achieved by using a fixed sweep rate from a zero field [51]. The $s$ and $w$, as functions of $H$, are plotted in Fig. 3 for various sweep rates. It indicates that the spacing $s$ can be manipulated from several to tens of micrometers. One of the other parameters affecting the width $w$ and space $s$ are chain length. This aspect can be altered by changing the width of the cell in the direction along the field. Temperature also has the same effect as decreasing the field strength. It is also noteworthy that the thickness of the cell can be used to control the number of layers of the one-dimensional periodic long-chain structures with some lateral offset between two neighboring layers.

\subsubsection{Perpendicular magnetic fields}

In contrast to needle-like chains in magnetic fluid films under parallel fields, cylindrical magnetic columns were formed in the film under perpendicular fields $[17,38,39,42]$. Through an observation for the time evolution of the formation of columns under a given field exceeding some critical field strength, the diameter $a$ of a column became larger and then saturated when the system reached an equilibrium state [42,52]. Ytreberg and McKay give a theoretical relationship between the saturated diameter and the applied magnetic field and the thickness of the film under perpendicular fields [52]. This predicted relationship could be fitted very well to the experimental data.

With an increase in the magnetic field strength, the number of columns increase and the size of columns in the film show a broadened distribution [17,39]. At this stage, columns are located randomly in the film. Each column acts in an isolated manner, and the interactive force between a column and its surrounding columns cannot be balanced. This structural pattern is called a disordered column pattern. Due to the nature of random distribution of magnetic columns in the magnetic fluid, any sort of detailed analysis for characterizing the structural pattern of these columns can hardly be carried out. Hence, currently published papers focus only on the agglomeration of magnetic particles.

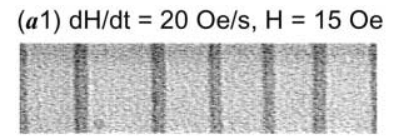

(a2) $\mathrm{dH} / \mathrm{dt}=20 \mathrm{Oe} / \mathrm{s}, \mathrm{H}=30 \mathrm{Oe}$

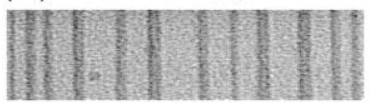

(a3) $\mathrm{dH} / \mathrm{dt}=20 \mathrm{Oe} / \mathrm{s}, \mathrm{H}=50 \mathrm{Oe}$

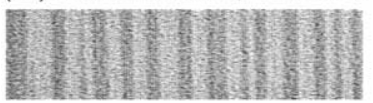

(a4) $\mathrm{dH} / \mathrm{dt}=20 \mathrm{Oe} / \mathrm{s}, \mathrm{H}=100 \mathrm{Oe}$

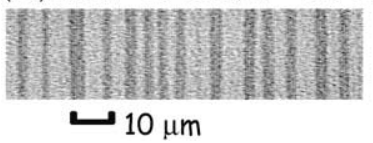

(b1) $\mathrm{H}=30 \mathrm{Oe}, \mathrm{dH} / \mathrm{dt}=10 \mathrm{Oe} / \mathrm{s}$

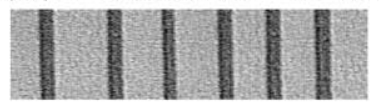

(b2) $\mathrm{H}=30 \mathrm{Oe}, \mathrm{dH} / \mathrm{dt}=20 \mathrm{Oe} / \mathrm{s}$
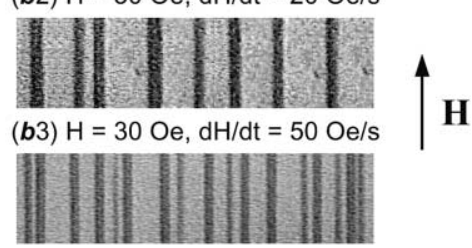

(b4) $\mathrm{H}=30 \mathrm{Oe}, \mathrm{dH} / \mathrm{dt}=100 \mathrm{Oe} / \mathrm{s}$

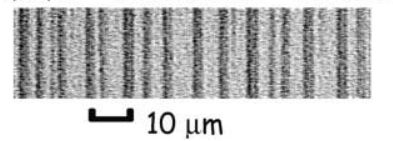

Fig. 2. Periodic long-chain structural patterns formed in a magnetic fluid film subjected to perpendicular fields exceeding a critical value, at: (a) different field strength with constant sweep rate or (b) different sweep rate with a given field strength [51]. The thickness of the film is $5.0 \mu \mathrm{m}$ and the concentration of the fluid is $22.4 \%$. 


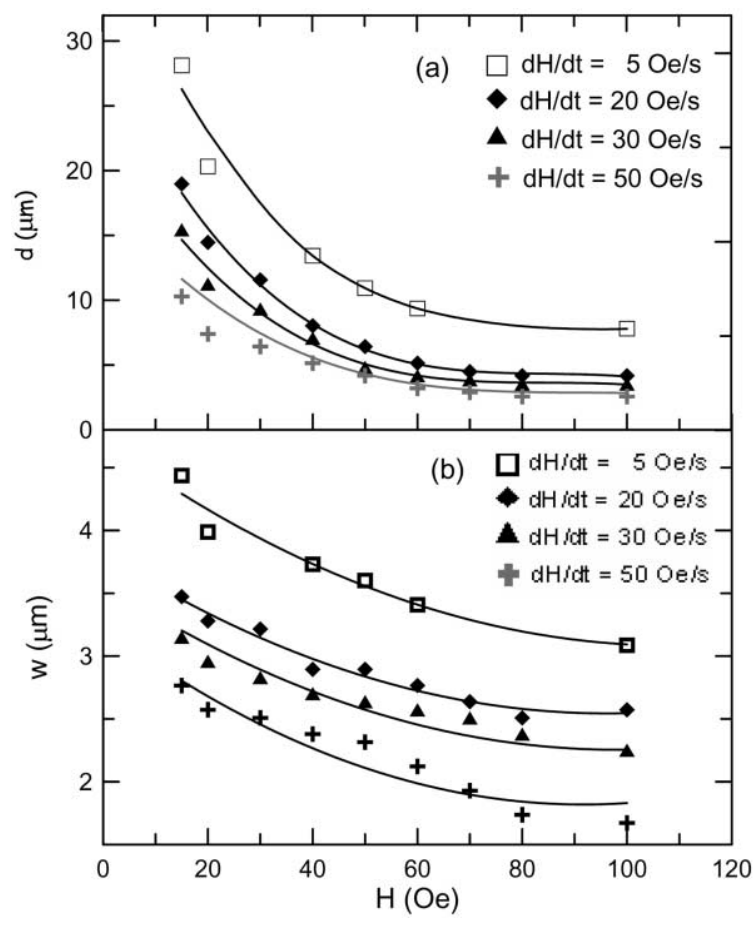

Fig. 3. Plot of (a) the distance between long chains $d$ and (b) the chain width $w$ as functions of field strength at various sweep rates [51]. The thickness of the film is $5.0 \mu \mathrm{m}$ and the concentration of the fluid is $22.4 \%$.

In our previous work [18], the structure in highly homogeneous magnetic fluid films evolved from a monodispersed state to a disordered one when the magnetic field $H$ was increased from zero to a critical value. If the $H$ was further increased, particles agglomerated to form new columns.
Then, a granular hexagonal ordered structure of columns was constructed, as shown in Fig. 4. When the $H$ kept increasing, more columns appeared. Due to the confinement by long-range grain boundaries, the columns were in grain contact with each other, and hence a labyrinthine pattern appeared in the magnetic fluid film. To our knowledge, this is the first time the two-dimensional ordered structure for the magnetic fluid film under external magnetic fields has been observed.

The ordered structure in a magnetic fluid film can be characterized by the averaged distance $d$ between two neighboring columns and the averaged diameter $a$ of columns. The averaged column distance $d$ is obtained by fast Fourier transformation of the image of an ordered structure. For the ordered state, the $d$ was found to decrease under a higher $H$, whereas $a$ remained almost constant. A typical relationship between $d$ and $H$ is plotted in Fig. 5, in which the magnetic field begins at zero and follows a certain sweep rate to a final magnetic field $H$ for each data point (referred to as a static process hereafter). In order to understand the physical mechanism governing the $d(H)$ curve, we developed a theoretical model and then fitted the predicted $d(H)$ curve to the experimental data [43]. In this model, magnetic columns are regarded as cylindrical magnetic dipoles (column dipoles). The magnetization is assumed to be uniform inside a column. Two interactions are taken into account for the energy of the two-dimensional magnetic dipole array: the attractive potential energy between the magnetic column and the external magnetic field, and the repulsive potential energy between two magnetic columns with parallel magnetization. The relationship between the column distance $d$ and the applied magnetic field $H$ was obtained by minimizing the total energy of the system with respect to column distance. The theoretical $d(H)$ curves exhibit a good consistency with the experimental $d(H)$, as
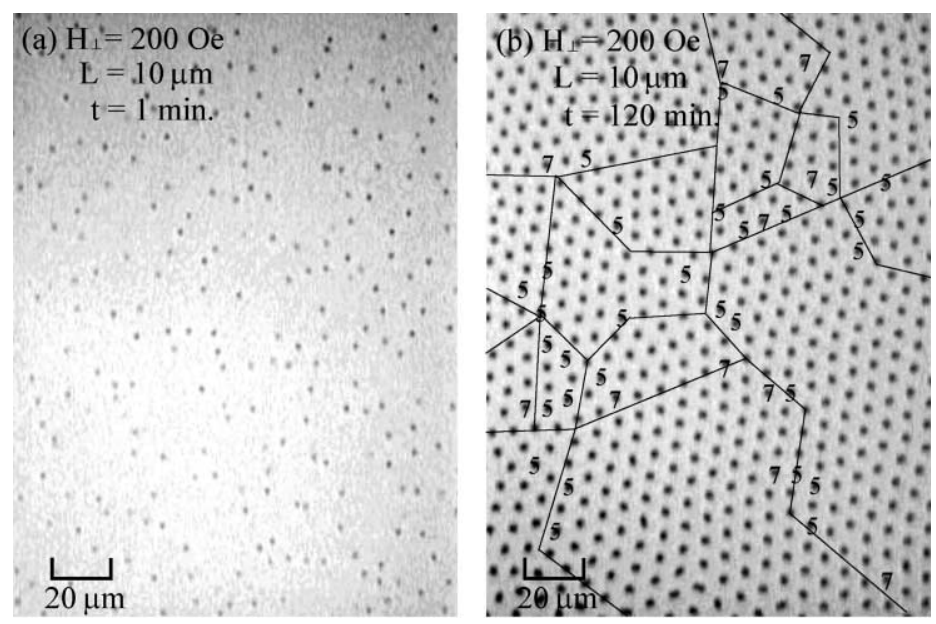

Fig. 4. (a) Initial ( $t=1 \mathrm{~min})$ disordered columns and (b) final $(t=120 \mathrm{~min})$ hexagonal column patterns when the magnetic fluid film of $10 \mu \mathrm{m}$ thickness cell was subjected to a perpendicular magnetic field, $H=200$ Oe [18]. Five-fold and seven-fold co-ordinated columns were observed along the grain boundaries in the final pattern. 


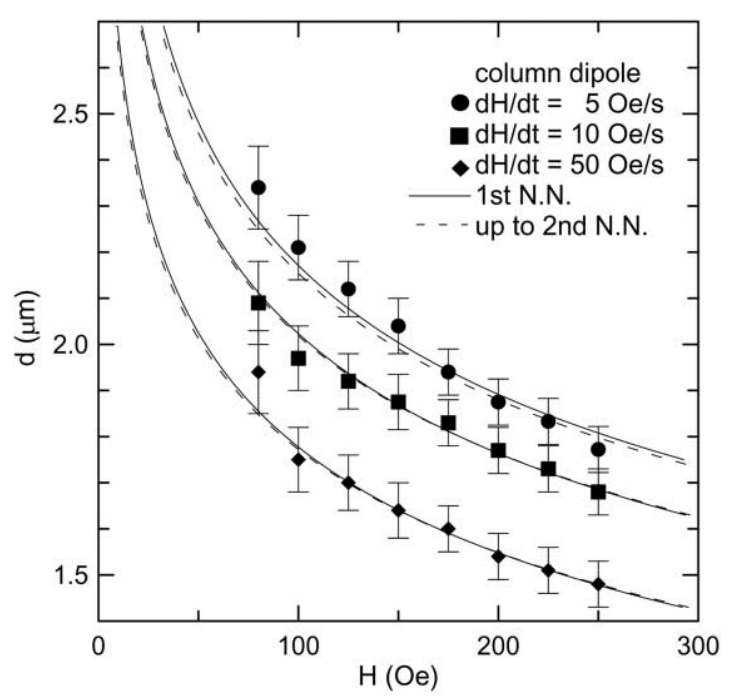

Fig. 5. Experimental column distance $d$ with error bar as a function of the applied magnetic field $H$ at three sweep rates $d H / d t=5,10$ and $50 \mathrm{Oe} / \mathrm{s}$ [53]. The solid and dashed lines are the theoretical $\mathrm{d}(H)$ curves from the column-dipole behavior taking into consideration the interaction between the first nearest neighboring columns (1st $\mathrm{MNN}$ ) and up to the second neighboring columns ( $2 \mathrm{nd} \mathrm{NN}$ ), respectively. The thickness of the film used here is $6.0 \mu \mathrm{m}$ and the concentration of the fluid is $19.4 \%$.

shown in Fig. 5. Our other reports indicate that the $d(H)$ curve can be shifted by adjusting the controlled parameters, such as the sweep rate $d H / d t$ of the applied field, the thickness $L$ of the film, the concentration $\phi$ of the fluid and temperature $T$ [31,54]. According to these experimental results, the range of $d$ can be manipulated within several micrometers.

Another interesting phenomenon observed for structure evolution in the magnetic fluid film under perpendicular magnetic fields is the occurrence of the labyrinthine pattern. It is suggested that the labyrinthine pattern can be mainly attributed to the existence of long-range grain boundaries in the granular hexagonal structure [18]. If the number of longrange grain boundaries is reduced, the pattern formation may be different. By improving the synthesis process of the magnetic fluid, we have synthesized high-quality magnetic fluids, capable of forming large-area hexagonal structures in the film under perpendicular fields, as shown in Fig. 6 [44,50]. The structure formation under a static process was then re-examined for a $6.2 \mu \mathrm{m}$-thick film composed of the high-quality magnetic fluid with a concentration of $10.9 \%$. When a lower $H$ 's $(<50$ Oe for this sample) was applied at $d H / d t=5 \mathrm{Oe} / \mathrm{s}$, a disordered column phase was observed. As $H \geq 50 \mathrm{Oe}$, the pattern evolved from a disordered column phase to the first-level hexagonal structure. When the $H$ became higher, the structural pattern went through a phase transition, in which a column split into two columns, and finally reached the second-level hexago-

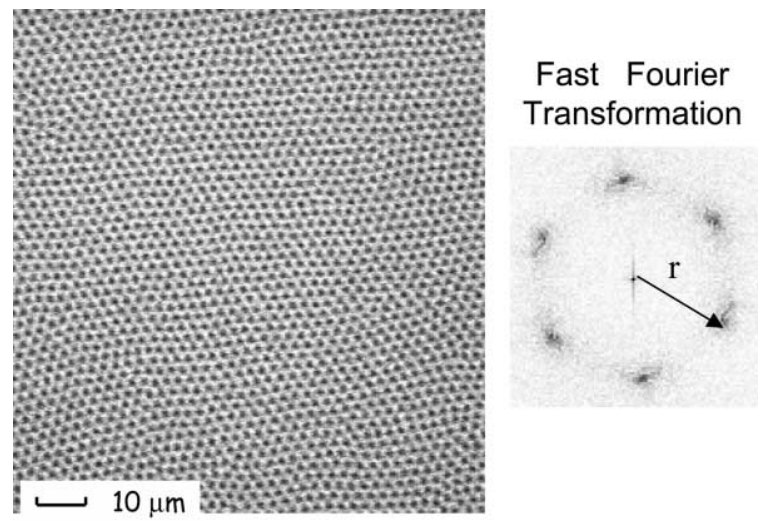

Fig. 6. A typical image of a large-area hexagonal structure of magnetic fluid thin film and its FFT transformation [53,54]. The column distance $d$ can be calculated from $d=2 \pi / \mathrm{r}$, where $r$ is the distance from the center to a vortex in the FFT image.

nal structure [44]. The same behavior was also found for the sweep rates of 2 and $10 \mathrm{Oe} / \mathrm{s}$. It is worth noting that the labyrinthine pattern appeared no more, instead, multiple states of ordered structures and a phase transition were observed for the magnetic fluid film under perpendicular magnetic fields.

The $d-H$ and the $a-H$ curves of the above structure evolution of static processes in magnetic fluid film under perpendicular magnetic fields with various sweep rates are plotted and shown in Fig. 7. For a lower sweep rate, say $5 \mathrm{Oe} / \mathrm{s}$, a plateau occurred from 50 to $180 \mathrm{Oe}$ in the $d-H$ and in the corresponding $a-H$ curve. This plateau refers to the firstlevel hexagonal ordered structure. When the $H$ was further increased over $180 \mathrm{Oe}, d$ and $a$ decreased monotonously due to the split of columns, followed by another plateau as the system reached the second-level hexagonal structure. However, for higher sweep rates, only one level of hexagonal structure was observed. The results in Fig. 7 also reveal that the diameter $a$ is reduced under a higher sweep rate. Based on our experience, the reduction in the column diameter can also be achieved by decreasing the film thickness, the temperature or by raising the fluid concentration [54].

To investigate the transition phase, the magnetic field $H$ was increased from 0 to $400 \mathrm{Oe}$ at $d H / d t=5 \mathrm{Oe} / \mathrm{s}$. The instant images in the transition region during the application of the field were taken. Some representative images are shown in Fig. 8. During the time shown by Fig. 8(a)-(d), the cross section of a column elongated form the original near circular shape. By increasing the magnetic field, the deformed column split into two columns as shown in Fig. 8(e). This process continued until all the columns split, then the next level hexagonal structural pattern was formed, as shown in Fig. 8(f). It should be noted that when the magnetic field was increased to a certain strength in the transition phase and remained constant at that strength, the columns 


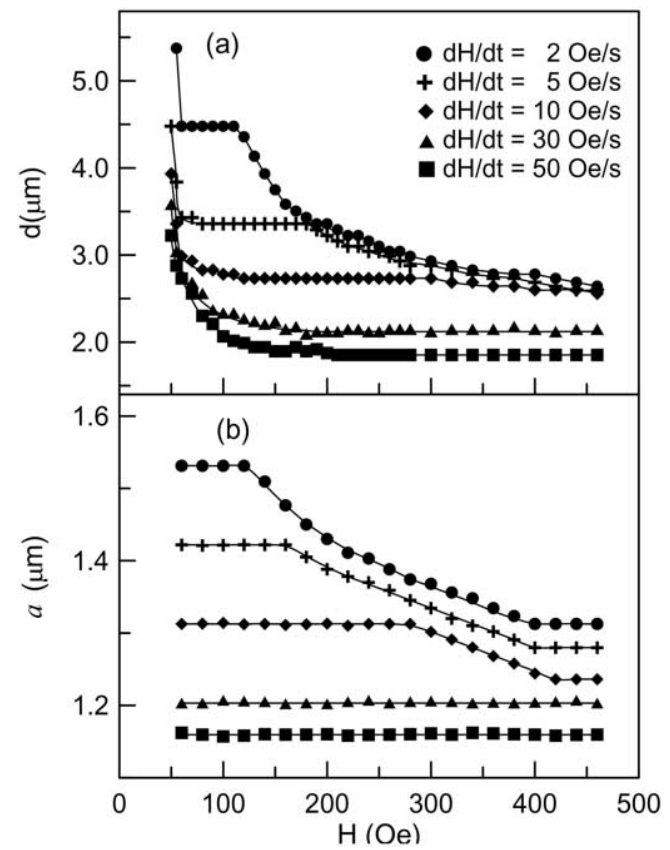

Fig. 7. (a) Plot of the distance between columns of hexagonal structure in magnetic fluid films as a function of field strength at various sweep rates, (b) plot of the corresponding hexagonal structure column size in a magnetic fluid film versus the field strength at various sweep rates [44]. The thickness of the film is $6.2 \mu \mathrm{m}$ and the concentration of the fluid is $10.9 \%$. continued to split for a while longer, and then the system reached an equilibrium state. Also, more particles in the surrounding liquid joined these two newly split columns and formed new columns during the transition.

The split of columns can be interpreted as follows. For a given column, there are two interactions relating to the split. One is the inter-column repulsive interaction $F_{\text {inter }}$. The other is the intra-column repulsive force $F_{\text {intra }}$, due to the domains inside the column. The $F_{\text {inter }}$ leads to a confinement of particles within the columns, whereas $F_{\text {intra }}$ causes the particles to separate from each other. When the magnetic field is increased, the $F_{\text {intra }}$ can be enhanced due to the fact that a better alignment of the magnetic particles at a higher field results in a larger intra-column repulsive force. When the $F_{\text {intra }}$ subdues the $F_{\text {inter }}$, the column splits into two columns.

So far, we have illustrated the rich phenomena of pattern formation in unsaturated magnetic fluid film under parallel or perpendicular magnetic fields. In actual applications, it may occur that the carrier of magnetic fluid escapes from the film, and the fluid inside the film becomes over-saturated. Some particles will then condense out of the fluid and form microdrops inside the film. In the next part, we introduce the pattern formation of an over-saturated magnetic microdrop system under external magnetic fields.

\subsection{Microdrops}

Remarkable shape evolution in magnetic fluid microdrops can be observed when a droplet of magnetic fluid is trapped (a) $\mathrm{H}=80 \mathrm{Oe}$

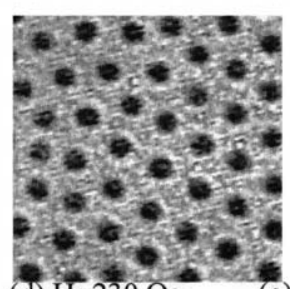

(d) $\mathrm{H}=230 \mathrm{Oe}$

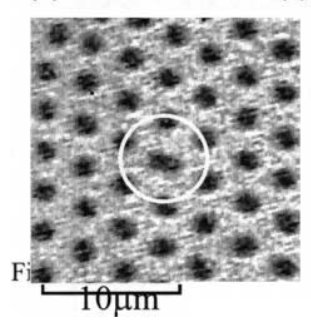

(b) $\mathrm{H}=220 \mathrm{Oe}$

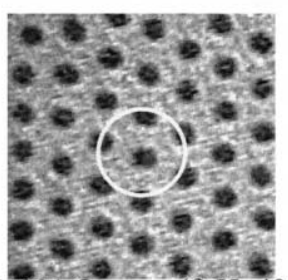

(e) $\mathrm{H}=231 \mathrm{Oe}$

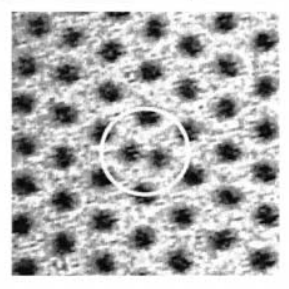

(c) $\mathrm{H}=229$ Oe
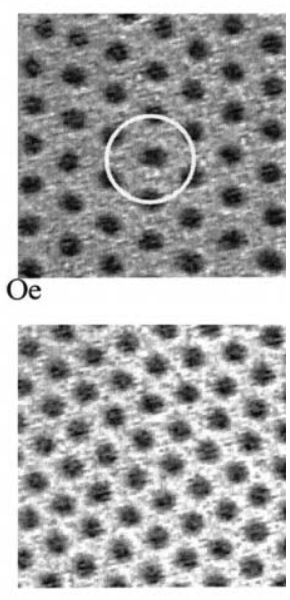

Fig. 8. Magnified photo images of the detailed transition phase of magnetic fluid thin film subjected to a perpendicular magnetic field at a low sweep rate of $5 \mathrm{Oe} / \mathrm{s}$ [44]. (a) First-level hexagonal structural pattern, (b) transition phase, (c) column elongation, (d) column necking, (e) column breaking, (f) second-level hexagonal structural pattern. In (b)-(e), a typical column breaking process of a particular column in the transition phase is circled. 

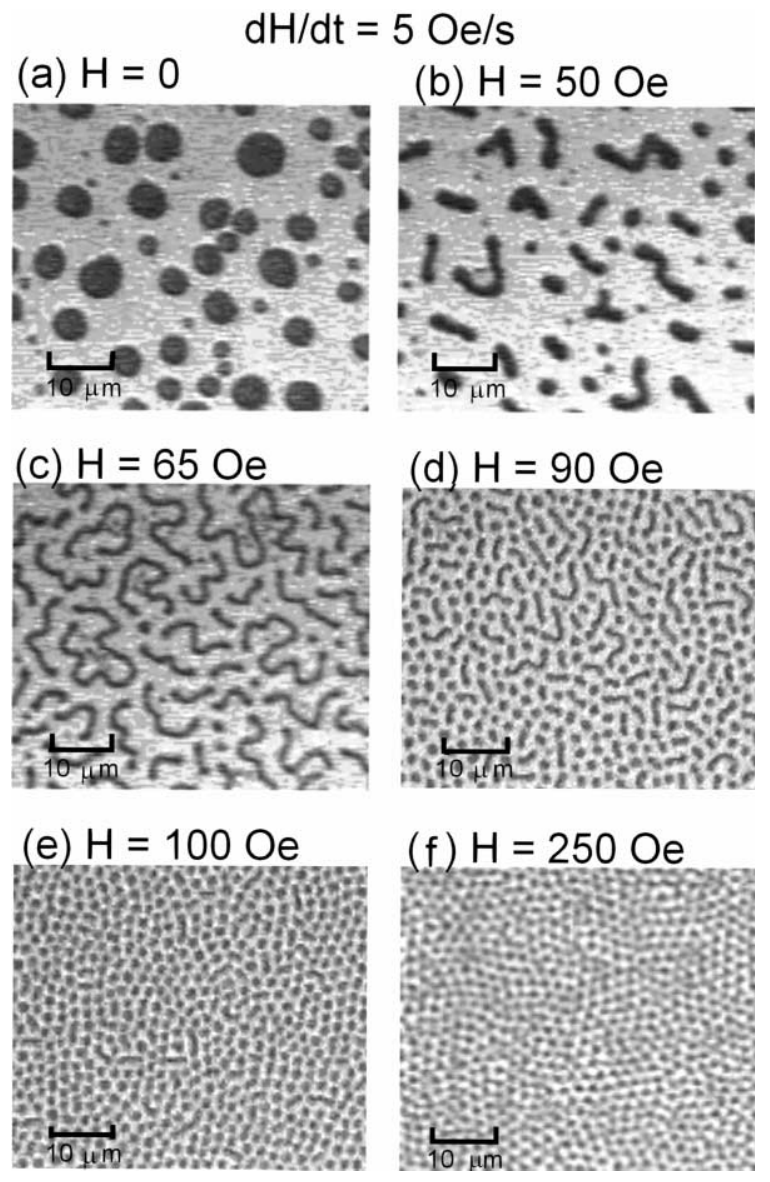

Fig. 9. Evolution of pattern formation of a magnetic fluid thin film containing magnetic microdrops subjected a perpendicular magnetic field at a low sweep rate of 5 Oe/s [59]. The thickness of the film is $10 \mu \mathrm{m}$.

between two horizontal glass plates under a perpendicular magnetic field. In the absence of an applied magnetic field, the equilibrium shape of the magnetic fluid drops is nearcircular, due to surface tension. When the magnetic field is applied, the shape is distorted, and the fingering phenomena occur. Tsebers et al. first reported that the original cylindrical drop evolves to a dumbbell and bends as the field strength increases over some thresholds [55]. Over another threshold, the dumbbell becomes labyrinthine. Langer et al. gave a theoretical description of the energetics of the system [56]. They indicated that during the fingering process, there is competition between droplet surface tension and bulkinduced magnetic dipole interaction. Also, they found that the fingering motion of each drop satisfies a constraint (fixed fluid volume) and is dominated by viscosity. Cebers and Drikis further carried out a theoretical study on the dynamics of the shape evolution of magnetic fluid drops [20]. They discovered that the contour of the circular shape of the drops was perturbed when a magnetic field
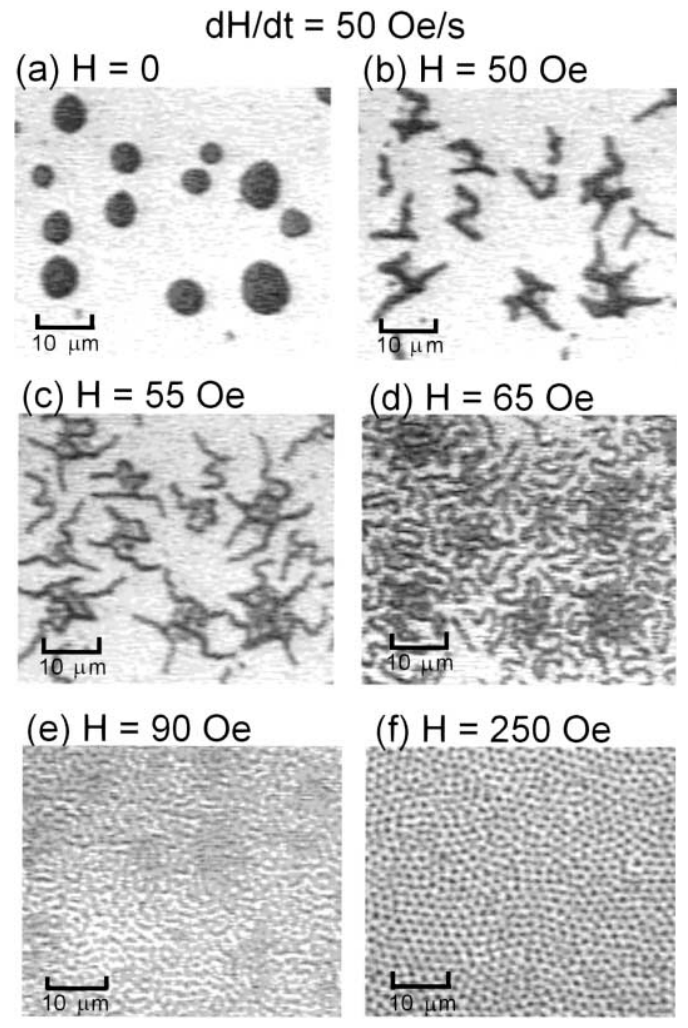

Fig. 10. Evolution of pattern formation of a magnetic fluid thin film containing magnetic microdrops subjected a perpendicular magnetic field at a high sweep rate of $50 \mathrm{Oe} / \mathrm{s}$ [59]. The thickness of the film is $10 \mu \mathrm{m}$.

was applied. This perturbed contour then acted as the initial state of the following fingering process. Some experimental results show that the degree of branching of the initially circular shapes depends not only on the magnetic field, but also on the rate of change of magnetic field strength $[57,58]$. It was also pointed out that the branched structure spreads without any further change in connectivity. This implies that the labyrinthine is a final state of a magnetic drop under a magnetic field.

After the success in synthesizing highly homogeneous magnetic fluid, we re-examined the structural evolution of magnetic microdrops under perpendicular magnetic fields. Figs. 9 and 10 show the evolutionary process of microdrops in an over-saturated magnetic fluid thin film [59]. Upon the application of a magnetic field, the circular drops of several micrometers evolve quickly to dumbbells (Fig. 9(b)) or to branched structures (Fig. 10(b)), depending on the field sweep rate. This observed result is in agreement with the prediction suggested by Dickstein [57]. At a lower sweep rate, magnetic particles within the drops are allowed to rearrange in such a way that a dumbbell shape can be formed. As the strength increases, the dumbbells elongate and bend (Fig. 9(c)). At a higher field strength, a 
(a)
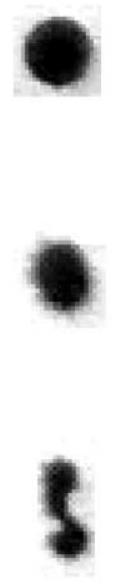

(b)
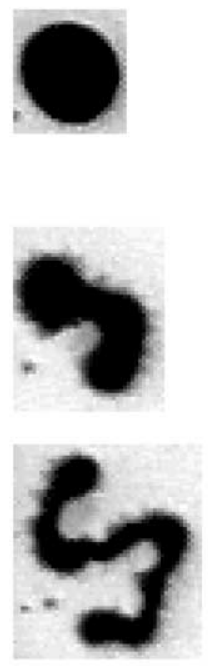

(c)
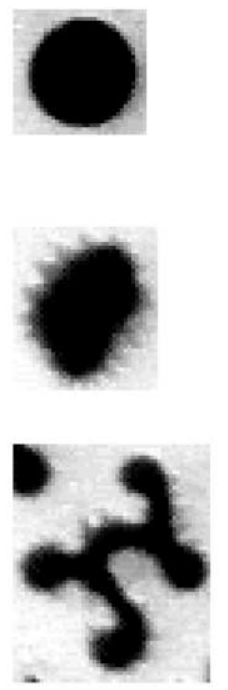

(d)

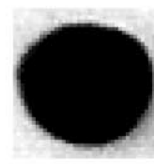

$$
\mathrm{H}=0 \mathrm{Oe}
$$
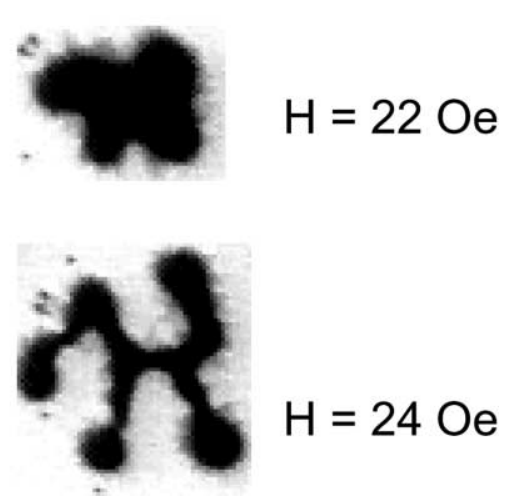

\section{$\mathrm{H}=24 \mathrm{Oe}$}

Fig. 11. Shape evolution of microdrops in the magnetic fluid thin film during the application of the perpendicular magnetic field [60]. Time increases downward. The $d H / d t$ is 1 Oe/s. The diameters of initially near circular microdrops through a to $d$ are 8.6, 12.8, 13.2 and 19.8 $\mu \mathrm{m}$.

labyrinthine structure forms in the film (Fig. 9(d)). If the strength is even higher, the labyrinthine structure becomes an equilibrium hexagonal structure (Fig. 9(e) and (f)). On the other hand, at a higher sweep rate, these drops evolve to a branched structure (Fig. 10(b) and (c)), to a labyrinthine pattern (Fig. 10(d) and (e)) and finally to an equilibrium hexagonal structure (Fig. 10(f)). It must be noted that the labyrinthine structure is indeed an intermediate state; this disagrees with the results given by prior reports [55-58].

The causes of the pattern formation are believed to be mainly the competition between surface tension, the bulkinduced magnetic dipole interactions and the attractive force from the external magnetic field. At the initial circular shape phase, the surface tension dominates. From the labyrinthine pattern to hexagonal structures, the magnetic dipole interaction dominates the surface tension, causing the breakup of connectivity of dumbbells or branched structure in the labyrinthine phase. This breakup repeats until the system reaches an equilibrium state, and the system becomes hexagonal. Here, the repulsive force, due to dipole interaction, and the attractive interaction, due to the external field, dominate. When sweeping the field back to the zero field state, the system changes from a hexagonal structure to a disordered column phase, and then to the initial circular form. Thus, the process of pattern formation in microdrops of magnetic fluid is irreversible.

In addition to the sweep rate, the size effect of magnetic fluid drops on fingering behavior has also been investigated [60]. The results are shown in Fig. 11. The upper images in Fig. 11(a)-(d) denote the initial contour of the microdrops under a zero field. The middle images represent the instant images of the corresponding initial contours during the application of the external magnetic field at $d H / d t=1 \mathrm{Oe} /$ s. In Fig. 11(a) and (b), both of the drops with representative diameters of 8.6 and $12.8 \mu \mathrm{m}$ evolved from a circular shape to a dumbbell structure. For the drops with diameters of 13.2 and $19.8 \mu \mathrm{m}$ (shown in Fig. 11(c) and (d)), the circular drops evolved into a branched, instead of a dumbbell, structure.

Since the surface tension minimizes the length of droplet contour and also a drop's surface energy, the energy by the surface tension of the dumbbell structure is lower than that of the branched structure. Thus, a dumbbell structure is formed when the surface tension dominates the fingering process, whereas a branched structure results when the bulk-induced magnetic dipole interaction suppresses the effect of the surface tension. By comparing the inference with the experimental results shown in Fig. 11, it can be concluded that the surface tension is a crucial factor in determining the shape evolution of smaller drops. On the other hand, the magnetic dipole interaction becomes more significant than the surface tension during the fingering process for larger drops.

The experimental results also imply that a critical diameter $a_{\mathrm{c}}$ for a given sweep rate, which differentiates these two evolved shapes, exits. Through a careful inspection, the $a_{\mathrm{c}}$ for $d H / d t=1 \mathrm{Oe} / \mathrm{s}$ was found to be approximately $13.0 \mu \mathrm{m}$. Furthermore, an empirical relationship between the $a_{\mathrm{c}}$ and the $d H / d t$ was obtained to be $a_{\mathrm{c}} \propto(d H /$ $d t)^{p}$ with $p \sim-0.14[60]$.

In summary, the agglomeration of magnetic particles in the magnetic fluid film under external magnetic fields is attributed to the thermodynamical instability of particles. In contrast to the disordered chain/column phase reported 
by other researchers, the one-dimensional/two-dimensional ordered structure was demonstrated for our high-quality magnetic fluid films under parallel/perpendicular magnetic fields. The characteristic spacing in the ordered structures can be manipulated within a range from sub-micrometers to tens of micrometers by adjusting controlled parameters such as the magnetic field, the sweep rate, the film thickness, the concentration of the fluid and the temperature. With these ordered structures, some significant optical properties are then generated and will be discussed in the next section.

\section{Optical properties of magnetic fluid films}

Due to its potential applications, the optical phenomena of magnetic fluids subjected to an external magnetic field have attracted sustained interest. Earlier investigations were focused on the magneto-optical effect, such as birefringence, transmittance and response time of the magnetic fluid films. In 1998, we first reported the magnetochromatic effect of homogeneous magnetic fluid films under a uniform perpendicular magnetic field [23]. This creates a new area for research on the magnetically induced optical phenomena of magnetic fluids. In this section, we give an overview on the magneto-optics and the magnetochromatics of magnetic fluids.

\subsection{Magneto-optics of magnetic fluids}

The earliest investigations on magneto-optics were carried out independently by Kerr, Majorana and CouttonMouton [61,62], but little further development was achieved until stable magnetic fluids became available in the 1960s. From then on, growing numbers of studies on the magnetooptics of magnetic fluids under external magnetic fields have been performed by many researchers because of the feasibility of the application of optical devices. In this field, the birefringence, transmittance and response time of magnetic fluids are the main subjects studied and are summarized as follows.

\subsubsection{Birefringence}

When a magnetic fluid thin film is subjected to an external magnetic field parallel to the plane of the film, the particles in the film agglomerate to form chains along the direction of the field. Thus, the magnetic fluids become optically anisotropic, and birefringence occurs as a light ray passing normally through the film. This anisotropy may originate from the shape anisotropy of ferrite particles [63], the needle-like chains $[21,36]$, the pre-existed magnetic chains [63], or the anisotropic interaction between the aligned ferrite particles [64]. According to these theoretical considerations, an elliptically polarized transmitted light resulted when a linearly polarized light was incident to the film. This phenomenon is known as the Cotton-Mouton (C-M) effect. These theories also predict that the transmission axis of the

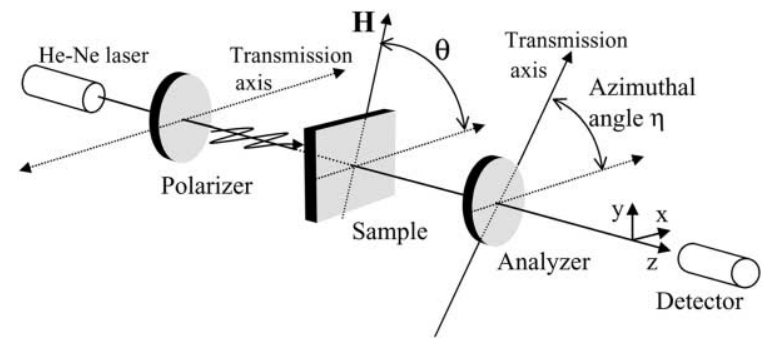

Fig. 12. Set-up of the experiment for investigating the CottonMouton effect of the magnetic fluid film [24,66].

transmitted light was rotated by an angle with respect to that of the incident light.

In almost all reported works, the C-M rotation of magnetic fluid films was indirectly calculated from the measured transmittance and the maximum and the minimum transmitted intensity through the films under a given magnetic field [21,22,36,65]. The experimental details were illustrated in Ref. [21]. With this method, the C-M rotations were investigated for the magnetic fluid film under various magnetic fields [36]. The temperature effect on the birefringence of the magnetic fluid film under parallel magnetic fields was investigated by Yusuf et al. [65]. It was observed that the birefringence is depressed at higher temperatures. A few years later, they reported the concentration and the wavelength dependent of the birefringence of magnetic fluid films [22]. The experimental results showed that the birefringence was enhanced for a sample of a higher concentration and that an optimum birefringence occurred at a wavelength around $500 \mathrm{~nm}$. These results were then explained in terms of the theory of dispersion of electromagnetic waves in the matter with the assumption that the density of charge carriers oscillating in a given direction is proportional to the number of particles orientated in that direction [22].

Notably, the reported C-M rotation is not obtained directly from the experimental data. In 1999, we developed a measurement of the azimuthal distribution of the transmitted intensity through a homogeneous magnetic fluid film under perpendicular magnetic fields [24]. This method affords a direct measurement of the $\mathrm{C}-\mathrm{M}$ rotation. The experimental set-up is shown in Fig. 12. A He-Ne laser beam (wavelength $=632.8 \mathrm{~nm}$ ) was linearly polarized by a polarizer and then was incident normally to the magnetic fluid film. The magnetic field here was generated by a pair of solenoids and applied to the film perpendicular to the propagation of the light. The angle $\theta$ between the transmission axis of the polarization of an incident light and the applied magnetic field can be adjusted by rotating the magnetic field. To examine the polarization of the transmitted light through the film, an analyzer was used to define the angular position of the maximum transmitted intensity. The azimuthal angle $\eta$, the angle between the two transmission axes of the polarizer and the analyzer, was determined by 


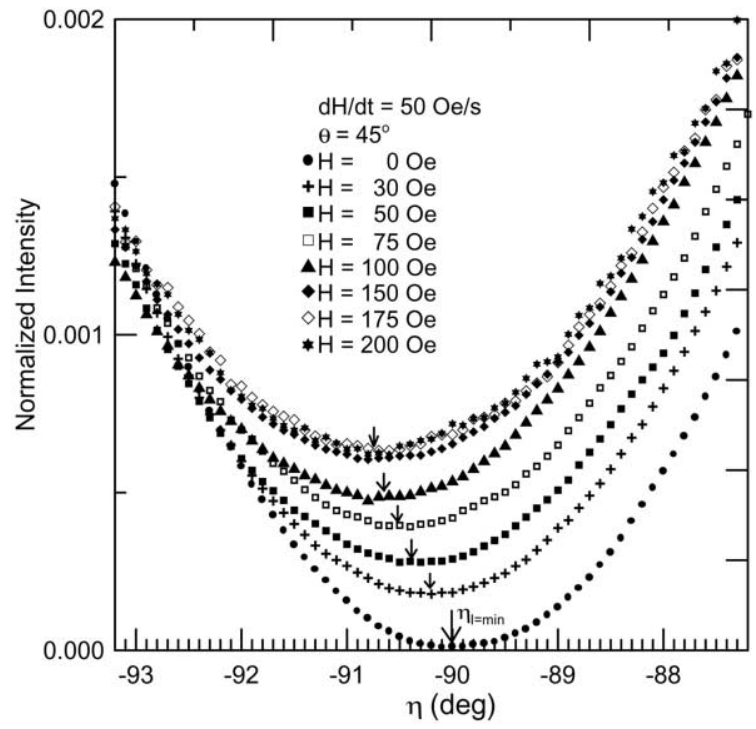

Fig. 13. Enlarged $I-\eta$ curves under various magnetic fields at $\theta=45^{\circ}$ [24]. The concentration of the magnetic fluid is $11.7 \%$ and the thickness of the film is $5 \mu \mathrm{m}$.

rotating the transmission axis of the analyzer with the aid of a stepping motor with a resolution of $0.01^{\circ}$. Finally, a PMT tube was used to detect the intensity of the transmitted light through the sample. It is noted that the instrument was aligned very carefully to obtain symmetric $I-\eta$ curves for various magnetic fields. From the $I$ - $\eta$ curves, the C-M rotation can be determined directly. In our work $[24,37,66]$, the magnetic field and the $\theta$-dependence of the $\mathrm{C}-\mathrm{M}$ rotations are investigated.

To examine the magnetic field dependence of C-M rotation, the $I-\eta$ curves were measured at $\theta=45^{\circ}$ with various $H$ 's. Under zero field, the symmetric $I-\eta$ curve exhibits a relative maximum intensity at $\eta_{I=\max }=0^{\circ}$ and zero intensity at $\eta_{I=\min }=90$ and $-90^{\circ}$. The symmetric $I-\eta$ curves under $H \neq 0$ were also obtained with a translational shift by a $\Delta \eta$ $\left(\equiv\left|\eta_{I=\min }\left(H, \theta=45^{\circ}\right)-\eta_{I=\min }\left(H=0, \theta=45^{\circ}\right)\right|\right.$ with respect to the zero field curve. This shift implies that the transmission axis of the transmitted light was rotated (i.e. C-M rotation) by an angle $\Delta \eta$ with respect to that of the incident light. To decide the $\Delta \eta$ precisely, the $I-\eta$ results at $\eta$ around $-90^{\circ}$ were enlarged and are shown in Fig. 13. The $\eta_{I=\min }$ is moved with the increase of the $H$ and then remains almost constant as $H>120$ Oe. This gives a magnetic-field-dependent C-M rotation, as shown in Fig. 14. In addition, the minimum intensity $I_{\text {min }}$ occurred at $\eta_{I=\min }$ is non-zero under $H \neq 0$. That $I_{\min } \neq 0$ suggests that the transmitted light is, not linearly, but elliptically polarized.

Since the birefringence of the magnetic fluid film is attributed to the formation of chains under parallel magnetic fields, the relationship between $\Delta \eta$ and the corresponding area ratio $r$ of chains to the liquid is plotted as the inset of Fig. 14. The linear relationship between $\Delta \eta$ and $r$ gives

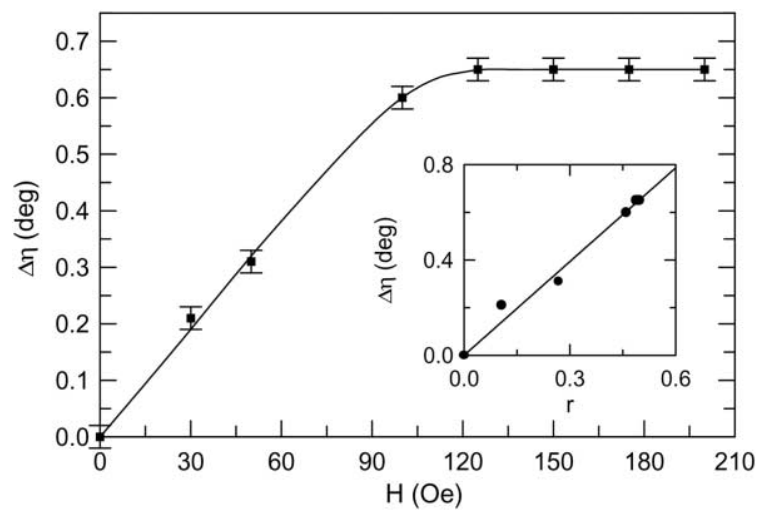

Fig. 14. C-M rotation angle $\Delta \eta$ versus the applied magnetic field [37]. The inset is the relationship between the $\Delta \eta$ and $r$, where $r$ is the area ratio of the long chains to the liquid carrier.

direct evidence that the birefringence of the magnetic fluid film is due to long chain formation.

$I-\eta$ curves for a given magnetic field and various $\theta$ 's were also used to investigate the $\theta$-dependent $\mathrm{C}-\mathrm{M}$ rotation of the magnetic fluid film [37]. Some typical $I-\eta$ curves are shown enlarged in Fig. 15. In Fig. 15(a) for $\theta=0$ or $90^{\circ}$, we found the minimum intensity $I_{\min }=0$ at the $\eta_{\min }=-90^{\circ}$. This indicates that there is no birefringence effect at $\theta=0$ and $90^{\circ}$. Increasing $\theta$ from 0 to $45^{\circ}$ in Fig. 15 (b) and (c), the $\eta_{\text {min }}$ gradually deviates from $-90^{\circ}$, and non-zero $I_{\min }$ occurs. If the $\theta$ is further increased to $90^{\circ}$, the $\eta_{\min }$ moves backward to $-90^{\circ}$. The $\Delta \eta$ as a function of $\theta$ is plotted in Fig. 16 .

The experimental data shown in Fig. 16 reveals that $\Delta \eta$ is a nearly symmetric function of $\theta$ with a maximum at $\theta$ close to $45^{\circ}$. In the conventional theoretical models for the birefringence of magnetic fluids [21,36,63,64], the film was regarded as a uniform birefringence slab, and the birefringence was due to the interaction between the incident light and the chains. Thus, $\Delta \eta$ was predicted to vary with $\theta, \zeta$ and $\delta$, where $\zeta$ is the ratio of the transmittances of the ordinary and the extraordinary lights, and $\delta$ denotes the phase difference between these two lights. The zero value of $\Delta \eta\left(=0^{\circ}\right)$ is then obtained when

$\theta=\left\{\cos ^{-1}[(\xi-1) /(2 \cos \delta+\xi+1)]\right\} / 2$

Some experimental results show that $\zeta$ is close to 1 $[21,63,64,67]$. Thus, that $\Delta \eta=0^{\circ}$ occurs at $\theta$ around $45^{\circ}$ via Eq. (1). This conclusion contradicts the experimental data shown in Fig. 16. Hence, conventional theories are not suitable for interpreting the C-M effect of magnetic fluid films.

To clarify the physical origin of the C-M rotation of the magnetic fluid film under parallel magnetic fields, we developed a two-phase model [37]. Phase I is the long chains and phase II is the liquid carrier. For phase I, the anisotropic shape of the chains causes a difference in the refractive indices of the lights polarized in the direction along (longitudinal) and perpendicular (transverse) to $H$ respectively. 


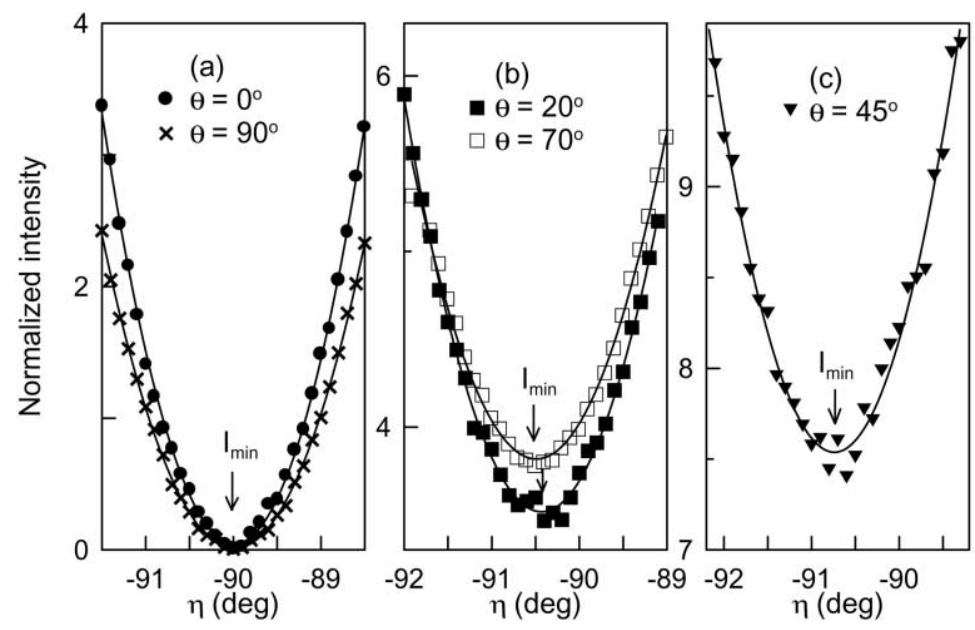

Fig. 15. The enlarged $I-\eta$ curves at $\eta$ around $-90^{\circ}$ under $H=150$ Oe and $d H / d t=50$ Oe at various $\theta$ 's are plotted in (a), (b) and (c) [66]. The concentration of the magnetic fluid is $11.7 \%$ and the thickness of the film is $5 \mu \mathrm{m}$.

Hence, to investigate the interplay between the magnetic chains and the incident light, the electric field of a linearly polarized incident light into phase I is decomposed into the longitudinal and the transverse electric fields. The transmitted electric fields of these two components are then calculated independently. For the phase II, the homogeneous liquid carrier exhibits an isotropic refractive index. Hence, the light transmitted through phase II remains line-

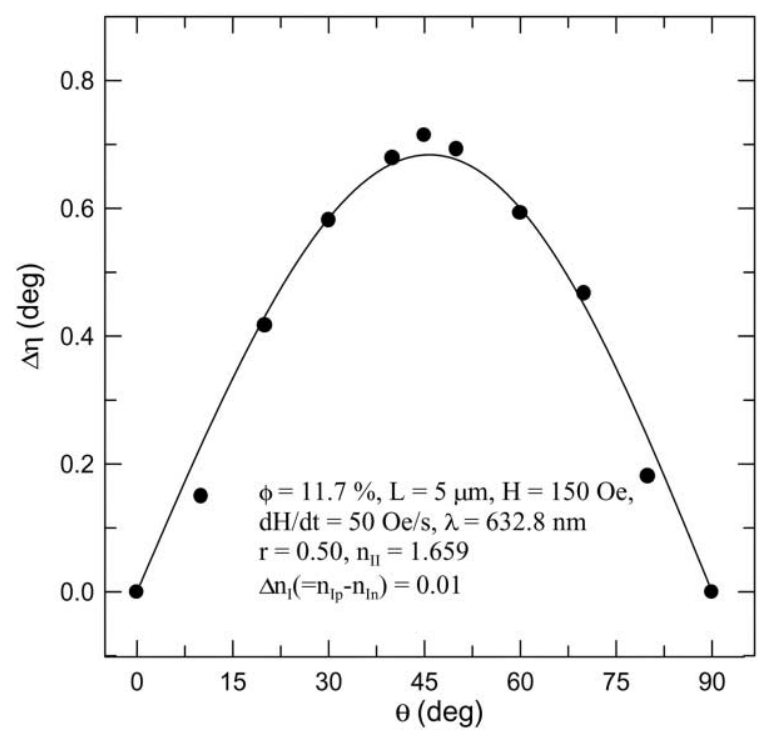

Fig. 16. Fitting between the experimental $\Delta \eta(\theta)$ data and the theoretical curve [66]. Dots denote experimental data, while the line represents the theoretical predictions obtained from the two-phase model. The $n_{I I}$ is the fitted refractive index of phase II (liquid carrier) and the $\Delta n_{I}$ denotes the difference between the refractive index $n_{I p}$ of the longitudinal electric field and that $n_{I n}$ of the transverse electric field of the incident light. arly polarized. By combining the transmitted electric fields through phases I and II, we obtain elliptical polarization for the transmitted light. Also, the C-M rotation is then calculated by finding the angle between the two transmission axes of the incident and the transmitted lights. The detailed derivation for the C-M rotation is developed in Ref. [37] and the analytic expression of $\Delta \eta$ is also given.

The experimental data shown in Fig. 16 are fitted to the theoretical $\Delta \eta(\theta)$ curve (denoted by a solid line). A good agreement between the experimental data and the theoretical prediction can be obtained. This strongly implies that the birefringence of the magnetic fluid films under parallel magnetic fields is due to the long chain formation in the film. Meanwhile, the interactions between the incident light and both the long chains and the liquid carrier must be taken into account to describe the $\mathrm{C}-\mathrm{M}$ rotation of the magnetic fluid films.

\subsubsection{Transmittance}

Due to the feasibility of optical devices such as the optical switch and the magnetic field sensor, the optical transmittance of magnetic fluid films are attracting the interest of a growing number of scientists, which is currently focused on the concentration, thickness and magnetic field and sweep rate-dependent transmittance. Martin et al. indicated that the transmittance of a magnetic fluid film is reduced substantially for fluids of a higher concentration [68]. Our recent experimental results show that the transmittance decreases exponentially with the increasing thickness of the film under a fixed magnetic field [69]. The attenuation distance of the incoming light through a diluted magnetic fluid thin film is then found to be hundreds of micrometers.

To study the effects of the magnetic field and the sweep rate on the transmittance, the transmitted intensities through a homogeneous magnetic fluid film under perpendicular magnetic fields were detected by one of the authors [70]. 


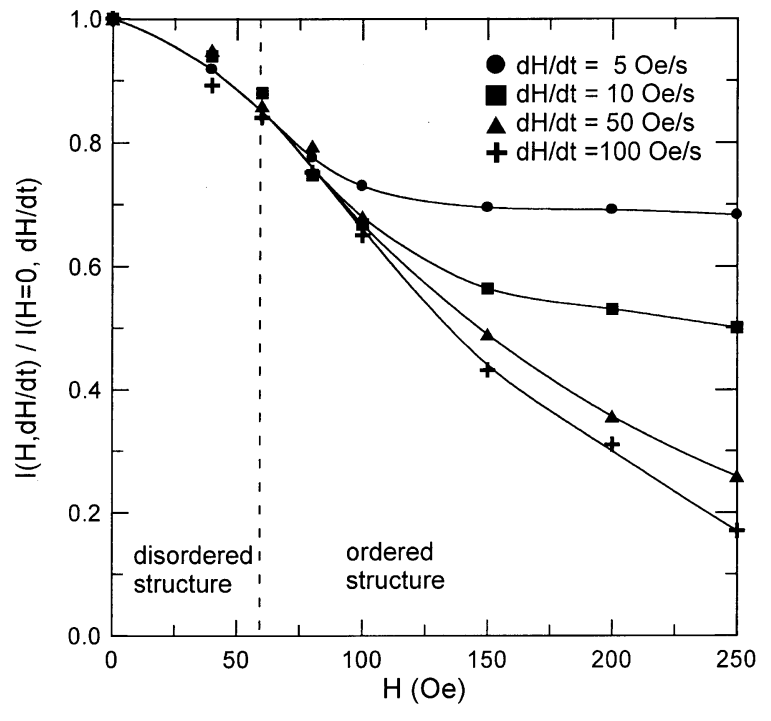

Fig. 17. Normalized intensity of an unpolarized He-Ne laser light as a function of field strength and its sweep rate for a magnetic fluid film of thickness $6 \mu \mathrm{m}$ subjected to a perpendicular magnetic field [70]. The concentration of the fluid is $27.8 \%$

In the experiment, an unpolarized $\mathrm{He}-\mathrm{Ne}$ laser light was incident perpendicularly to the film and the transmitted intensity was probed in the direction of light propagation by using a photomultiplier tube and a monochromator. The relative intensities of the transmitted light as a function of field strength for various sweep rates are shown in Fig. 17. All the intensities are normalized with respect to zero field intensity. The intensity decreases as the field strength increases. At low fields $(<60 \mathrm{Oe})$, the intensity curves are almost independent of the sweep rate. Contrarily, at higher fields ( $>60 \mathrm{Oe}$ ), the curves become sweep rate dependent. It shows that the intensity curves separate into two regions; one is sweep rate dependent and the other is independent of sweep rate.

Through an investigation on the structures in the film under the corresponding field strength and sweep rate, it was found that the disordered column phase occurred under $H<60 \mathrm{Oe}$ and the hexagonal ordered structure resulted as $H \geq 60 \mathrm{Oe}$. This suggests that structure formation plays an important role in the magnetically modulated transmittance of the magnetic fluid film under perpendicular fields. Also, the effect of the sweep rate becomes significant to the transmittance of the film exhibiting hexagonal ordered structures.

On the other hand, the transmittance generated by the birefringence of the magnetic fluid film under magnetic fields parallel to the plane of the film was examined [51,70]. The experimental set-up is shown in Fig. 12. Here, $\theta$ is set at $45^{\circ}$ and $\eta$ is at $-90^{\circ}$. Under a zero field, the transmitted intensity is zero due to the orthognality of the two transmission axes of the polarizer and the analyzer.

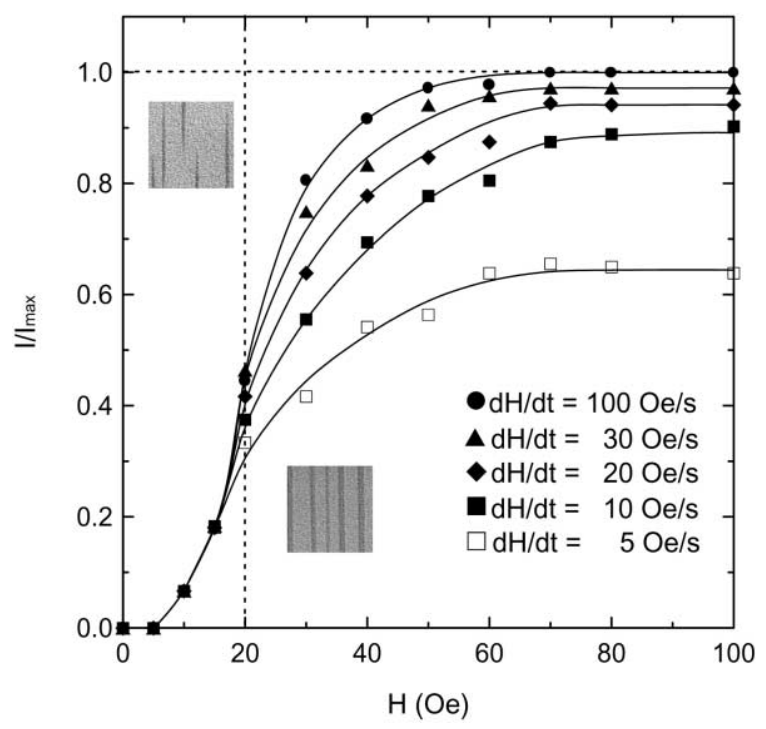

Fig. 18. Normalized intensity of a polarized He-Ne laser light as a function of parallel field strength and its sweep rate with the insets of typical structure patterns, for a magnetic fluid film of thickness $5 \mu \mathrm{m}$ oriented with an angle $\theta=45^{\circ}$ between the direction of the field and the axis of polarizer [70]. The concentration of the fluid is $22.4 \%$.

When a magnetic field is applied along the film surface, chains are formed and a birefringence is then induced. The birefringence leads to an elliptically polarized transmitted light. Thus, non-zero intensity can be detected as the transmitted light passes through the analyzer. Fig. 18 gives the normalized intensity of the transmitted light measured directly after the analyzer as functions of the field strength for various sweep rates. In contrast to the reduction in the transmitted intensity with the increasing field strength for the magnetic fluid film under perpendicular magnetic fields, these curves show that transmitted intensity increases under higher field strength. Through a close inspection, the intensity initially increases as the field strength increases $(<20 \mathrm{Oe})$, independent of the sweep rate. When the field strength is further increased $(>20 \mathrm{Oe})$, the intensity tends to saturate. The intensity saturates at a relatively low field strength for a higher sweep rate. These curves also show that a critical value $(\approx 20 \mathrm{Oe})$ divides these curves into two regions; one is independent of sweep rate and the other is sweep rate dependent.

Since the structural patterns in the magnetic fluid film are dominant to the transmittance, we then investigated the structural pattern in magnetic fluid films under parallel magnetic fields. It was found that a transition from disordered short chains to a nearly periodic long-chain structure occurred at $20 \mathrm{Oe}$, which coincides with the critical field in Fig. 18. The typical structures of short chains and periodic long chains corresponding to these regions are shown as the insets of Fig. 18. It is worth noting that the magnetically 


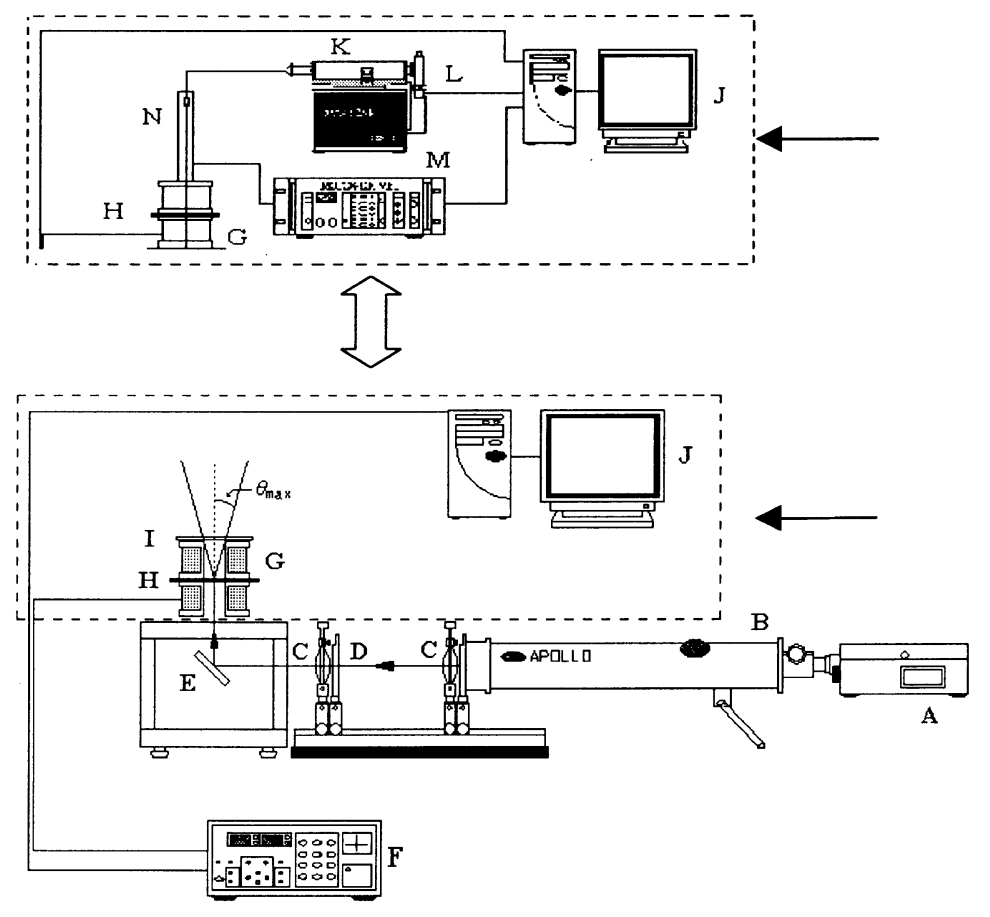

Fig. 19. Experimental set-ups [45]. (a) For the observation of hexagonal structure patterns in magnetic fluid thin films subjected to a magnetic field perpendicular to the plane of film. A: CCD video camera; B: PC; C: microscope; D: solenoid; E: magnetic fluid thin film; and F: current source. (b) For the observation of magnetochromatic effect of magnetic fluid thin films subjected to a magnetic field perpendicular to the plane of film. A: white light source; B: telescope; C: lens; D: aperture; E: mirror; F: current source; G: solenoid; H: magnetic fluid film; I: photo film; J: PC; K: monochromator; L: photomultiplier tube; M: stepping motor controller; and N: stepping motor with optical fiber.

modulated transmittance shown in Figs. 17 and 18 suggests a possibility for the development of optical devices such as optical switches.

\subsubsection{Response time}

For the development of optical devices by utilizing the magnetically modulated transmittance of the magnetic fluid film, it is necessary to know the corresponding optical response time. To probe the response [71], we used a He$\mathrm{Ne}$ laser to emit light incident normally to the magnetic fluid film under perpendicular magnetic fields. The transmitted intensity through the film was detected by using a photodiode with a response time of several nanoseconds. The signal detected by the photodiode was then converted to a voltage signal via an analog-digital converter. Thus, the time evolution of the transmittance through the magnetic fluid film can be measured.

The magnetic field was provided by a solenoid. By changing the current applied to the solenoid, the magnetic field was varied. The time constant of charging or discharging the current is tens of microseconds by using a constant current source (Keithley 228). It was found that the transmitted intensity $I$ through the magnetic fluid film increases to a saturated value $I_{0}$ when the magnetic field is reduced suddenly from a higher value to a lower one. The time evolution in the transmitted intensity follows $I(t)=I_{\mathrm{o}}[1-$ $\exp (-t / \tau)]$, where $\tau$ is the response time of transmittance and the time is counted from the moment at which the magnetic field changes. The experimental results show that $\tau$ is about tens of milliseconds, which was also observed by Martin et al. [68] by using a similar experimental method.

\subsection{Magnetochromatics}

When a homogeneous magnetic fluid thin film is subjected to perpendicular magnetic field, initial disordered columns form. At a critical field strength, an equilibrium two-dimensional hexagonal structure forms with columns occupying lattice vertices [44,72,73]. The distance $d$ between two columns was found to be several micrometers $[31,54]$. Thus, the hexagonal structure may act as a twodimensional optical grating which is capable of diffracting an incoming visible light. The set-up illustrated in Fig. 19 was used to demonstrate magnetochromatic effects resulting from the diffraction of light through the ordered structure $[23,73]$.

In the experiment, a white light was emitted from a light source. After passing through the lens, the white light became parallel and was then incident normal to the film. 


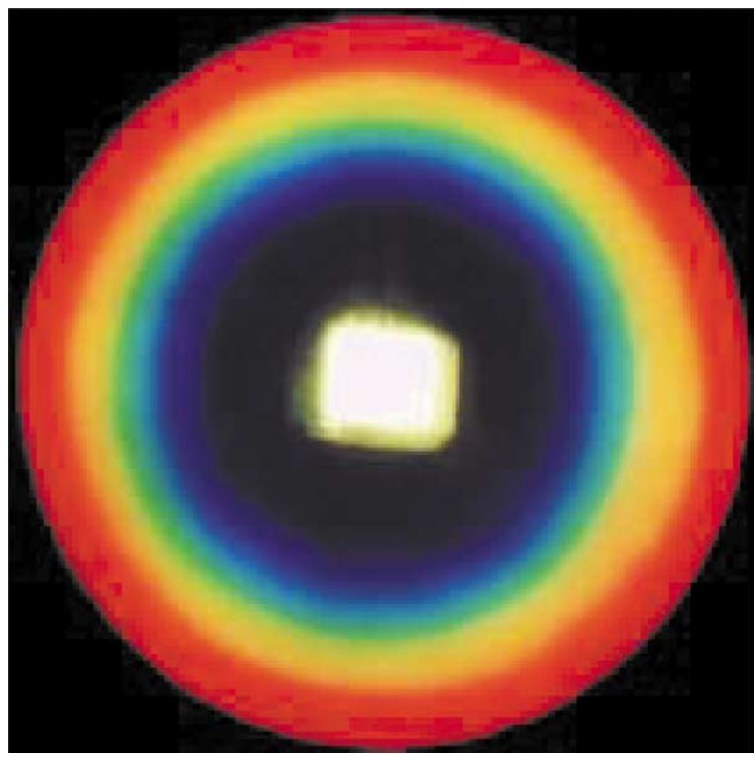

Fig. 20. Chromatic ring resulting from the diffraction and interference as the parallel beam of white light passing through the magnetic fluid film subjected to perpendicular magnetic fields [45].

The solenoids were well arranged to provide uniform magnetic fields perpendicular to the plane of the film. As the parallel light was transmitted through the film, it was directly exposed onto Kodak color film, placed above the solenoid as shown in set-up I of Fig. 19. To determine the distribution of the chromatic rings, set-up II was used to replace set-up I of Fig. 19. In set-up II, an optical fiber connected to a spectrometer was used to receive the diffracted light. A stepping motor with resolution of $0.01^{\circ}$ was used to control the angle of the optical fiber with respect to the vertical.

Fig. 20 is a typical chromatic ring that resulted from the passage of a white light through the hexagonal structure in a magnetic fluid film [31]. The circular shape of the chromatic ring is due to the misorientation of hexagonal lattices. The central area in the chromatic ring is the zero order principal maximum of the diffraction pattern. Since the incoming white light consists of a mixture of all visible lights, a bright white spot appears at the center. In the first order principal maximum of diffraction pattern, the incoming white light is dispersed. The spatial distribution of colors in the chromatic ring depends on the wavelength of light; red appears on the outmost ring, and, successively, orange, yellow, green, blue, and violet. This phenomenon can be well explained by the grating equation $d \sin \theta=\kappa \lambda$, where $d$ is the column distance, $\theta$ the angle between the outgoing color light beam and the direction normal to the film which was the vertical in this set-up, $\lambda$ the wavelength of the light, and $\kappa=0,1,2, \ldots$ By giving a fixed $d$, a longer wavelength leads to a larger $\theta$ and also a bigger circular ring as $\kappa=1$.

As indicated in Section 2.1.2, the column distance $d$ in the ordered hexagonal structure can be adjusted by changing the parameters, such as the field strength, the sweep rate, the thickness of the film, and the volume concentration, of homogeneous magnetic fluids. This implies that the magnetochromatic ring can be well controlled by altering these parameters. The flexibility of the magnetochromatics for the magnetic fluid films was then demonstrated and shown in Ref. [45]. It was observed that the chromatic ring becomes larger with increasing field strength, sweep rate and concentration, or decreasing thickness of the film.

The spatial diffraction patterns were then probed by using the set-up II in Fig. 19 to find the angle $\theta_{\text {exp }}$ corresponding to the first order maximum intensity of the diffracted light of a given wavelength, say $\lambda=535 \mathrm{~nm}$. Table 1 gives a summary of the results calculated from the structures and measured from the corresponding magnetochromatic rings under various control parameters. Table 1 indicates that the calculated diffraction angles $\theta_{\text {cal }}$ at wavelength $\lambda=535 \mathrm{~nm}$ based on the grating equation perfectly agree with those obtained from the measurement of the diffraction patterns.

Table 1

The column distance $d$ and grating angle $\theta$, including the conditions of $H, d H / d t, \phi$ and $L$ [45]. The $\theta_{\text {cal }}$, is calculated via the relation $\theta_{\text {cal }}=\sin ^{-1}$ $(\lambda / d)$ with $\lambda$ being $535 \mathrm{~nm}$, while the $\theta_{\exp }$ is obtained experimentally

\begin{tabular}{llllllll}
\hline No. & $L(\mu \mathrm{m})$ & $\phi(\%)$ & $H(\mathrm{Oe})$ & $d H / d t(\mathrm{Oe} / \mathrm{s})$ & $d(\mu \mathrm{m})$ & $\theta_{\text {cal }}\left({ }^{\circ}\right)$ & $\theta_{\exp }\left({ }^{\circ}\right)$ \\
\hline 1 & 6 & 21.69 & 50 & 5 & 2.34 & 13.22 & 13.20 \\
2 & 6 & 21.69 & 100 & 5 & 2.26 & 13.69 & 13.61 \\
3 & 6 & 21.69 & 200 & 5 & 1.64 & 19.04 & 19.03 \\
4 & 6 & 21.69 & 100 & 5 & 2.26 & 13.69 & 13.61 \\
5 & 6 & 21.69 & 100 & 20 & 1.64 & 19.04 & 19.11 \\
6 & 6 & 21.69 & 100 & 50 & 2.00 & 23.20 & 23.90 \\
7 & 4 & 21.69 & 100 & 5 & 2.26 & 15.51 & 13.69 \\
8 & 6 & 21.69 & 100 & 5 & 2.37 & 13.02 & 13.61 \\
9 & 8 & 21.69 & 100 & 5 & 2.35 & 13.69 & 13.16 \\
10 & 6 & 21.69 & 100 & 5 & 2.51 & 13.03 \\
11 & 6 & 19.67 & 100 & 5 & 5 & 13.61 \\
12 & 6 & 17.47 & 100 & & & 13.25 \\
\hline
\end{tabular}


This reveals that the hexagonal structure in the magnetic fluid film under perpendicular magnetic fields can act as a two-dimensional tunable grating.

In summary, the physical origin of the birefringence for the magnetic fluid film under parallel magnetic fields is confirmed to be the formation of long chains. The variation in the structural pattern in the film under various magnetic fields leads to the magnetically modulated optical transmittance. Besides, the hexagonal structure generates the magnetochromatic effect as a white light passes through it. The properties show a feasibility of application of magnetic fluids in optical devices, such as optical switch, filter or tunable grating.

\section{Conclusion}

The structures of the agglomeration of the magnetic nanoparticles have been reviewed in this report. Through the tremendous research on ferrofluids in recent years, the investigators found a variety of structural patterns of chains/columns in the ferrofluid thin film under magnetic fields. Among these structures, the most spectacular pattern is the ordered structure of magnetic chains/columns formed in the homogeneous ferrofluid thin film. It was further discovered that the characteristic spacing in the ordered structure can be manipulated within a range from submicrometers to tens of micrometers by adjusting the controlled parameters. The ordered structures generate the abundant magneto-optic effects, such as birefringence, field-dependent transmittance, etc. for the ferrofluid thin films. It is worth mentioning that the ordered hexagonal structure of columns in the ferrofluid thin film under perpendicular magnetic fields results in the novel magnetochromatics. This phenomenon implies that the ferrofluid thin film with the ordered hexagonal structure acts as a two-dimensional tunable grating.

Although numerous work has been done in the investigation of the fruitful properties of the ferrofluids under magnetic fields, there still exists a vast unexplored territory of the research in the related fields, including the materials, the fundamental mechanism and the applications, waiting for the scientists to pioneer. First of all, the ferrofluid which was composed of the magnetic nanoparticles, surfactant and carrier may be modulated by each component to create some completely new materials to enhance the Cotton-Mouton rotation and to sharpen the transition of the transmittance and other similar optical quantities. For the point of view of certain applications, the domain size of the hexagonal structure of the magnetic agglomeration is rather concerned. It is worth further investigation in the future. Considering the mechanical applications, a magnetic field and temperature stable ferrofluid is invariably the common research target for the scientists. In general, the smaller the $H_{\mathrm{h}}$ of the ferrofluid, the more widespread their application, here $H_{\mathrm{h}}$ denotes the required field strength to achieve the ordered structure in the magnetic fluid film. Hence the material with $H_{\mathrm{h}}$ less than $50 \mathrm{Oe}$ is suitable for applications.

Fundamentally, the present research seems to lack a strict description of the mechanism of formation of the column/ chain, the structure of the intra column/chain, etc. These are also important prospects for further research in the area.

As reported in this review article, significant optical properties of the ferrofluid forecast a promising opportunity for the wide application in optical devices, such as optical switches, filters, or optical lens, and some other advanced optical components. As one can see, these explorations require integrated co-operation among aspects of specialists in the future. It is believed that this spark of wisdom will have many commercial impacts.

We are looking forward to the ferrofluid playing an important role in both fundamental research and applications in the coming century.

\section{References}

[1] F. Reinitz, Monatshefte fur Chemie 9 (1888) 421.

[2] O. Lehmann, Z. Physik Chem. 4 (1889) 462.

[3] D. Friedel, Ann. Physique 18 (1922) 273.

[4] W. Kuhn, A. Ramel, D.H. Walters, G. Ebner, H.J. Kuhn, Fortschr. Hochpolym. -Forsch. 1 (1960) 540.

[5] J.P. Chrichley, Progr. Polymer Sci. 2 (1970) 1.

[6] P.G. de Gennes, Scaling Concepts in Polymer Physics, Cornell, Ithaca, NY, 1979.

[7] G. Jannik, J. Des Cloizeaux, Polymers in Solution, Oxford University, London, 1992.

[8] E.E.D. Chidsey, R.W. Murray, Science 231 (1986) 25.

[9] W. Krutschmer, L.D. Lamb, K. Fostiropoulos, D.R. Huffman, Nature 347 (1990) 10.

[10] F.J. Arriagada, K. Osse-Asave, J. Coll. Inter. Sci. 170 (1995) 8.

[11] National Science Foundation report: Nanostructure Science and Technology: R \& D Status and Trends in Nanoparticles, Nanostructured Materials, and Nanodevices. (1999).

[12] US Pat. No. 3215572.

[13] US Pat. No. 3917538.

[14] US Pat. No. 4019994.

[15] R.E. Rosensweig, Sci. Am. 247 (1982) 136.

[16] Susamu Taketomi, Hiromasa Takahashi, Nobuyuki Inaba, Hideki Miyajima, J. Phys. Soc. Jap. 60 (1991) 1689.

[17] Hao Wang, Yun Zhu, C. Boyd, Weili Luo, A. Cebers, R.E. Rosensweig, Phys. Rev. Lett. 72 (1994) 1929.

[18] Chin-Yih Hong, H.E. Horng, I.J. Jang, C.J. Hsu, Y.D. Yao, H.C. Yang, J. Appl. Phys. 81 (1997) 4275.

[19] R.E. Rosensweig, J. Magn. Magn. Mater. 201 (1999) 1.

[20] I. Drikis, A. Cebers, J. Magn. Magn. Mater. 201 (1999) 339.

[21] A. Peterlin, H.A. Stuart, Z. Phys. 112 (1939) 129.

[22] N.A. Yusuf, A. Ramadan, H. Abu-Safia, J. Magn. Magn. Mater. 184 (1998) 75.

[23] Herng-Er Horng, Chin-Yih Hong, Wai Bong Yeung, HongChang Yang, Appl. Opt. 37 (1998) 2674.

[24] H.E. Yang, Chin-Yih Hong, H.C. Yang, I.J. Jang, S.Y. Yang, 
J.M. Wu, S.L. Lee, F.C. Kuo, J. Magn. Magn. Mater. 201 (1999) 215.

[25] R.E. Rosensweig, Nature 210 (1966) 613.

[26] M.P. Perry, T.B. Jones, J. Appl. Phys. 49 (1976) 2334.

[27] L. Mellilo, K. Ray, J. Audio Eng. Soc., (USA) 29 (1981) 132.

[28] H. Yamaguchi, I. Kobori, N. Kobayashi, J. Magn. Magn. Mater. 201 (1999) 260.

[29] Y.S. Kim, K. Nakatsuka, T. Fujita, T. Atarashi, J. Magn. Magn. Mater. 201 (1999) 361.

[30] Susamu Taketomi, US pattern 4812767.

[31] Hong, Chin-Yih Rex, Horng,Herng-Er, Yang,Hong-Chang, Yeung,Wai Bong, U.S. Pat. No. 5948321.

[32] Jong-Wook Seo, Sang Ju Park, Kyeong Oh Jang, J. Appl. Phys. 85 (1999) 5956.

[33] R.S. Newbower, Proc. 1992 Biomedical Symp., San Diego.

[34] F. Charles, J. Hayes, Colloid Inter. Sci. 52 (1975) 239.

[35] Nihad A. Yusuf, J. Phys. D: Appl. Phys. 22 (1989) 1916.

[36] S. Taketomi, S. Ogawa, H. Miyajima, S. Chikazumi, IEEE Trans. Magn. 4 (1989) 384.

[37] S.Y. Yang, W.S. Tse, H.E. Horng, H.C. Yang, Chin-Yih Hong, J. Magn. Magn. Mater. 226-230 (2001) 2035.

[38] G.A. Jones, H. Niedoba, J. Magn. Magn. Mater. 73 (1988) 33.

[39] G.A. Jones, A. Moman, IEEE Trans. Magn. 26 (1990) 1849.

[40] J. Liu, E.M. Lawrence, A. Wu, M.L. Ivey, G.A. Flores, K. Javier, J. Bibette, J. Richard, Phys. Rev. Lett. 74 (1995) 2828.

[41] A.T. Skjetorp, Phys. Rev. Lett. 51 (1983) 2306.

[42] J. Cernak, P. Macko, J. Magn. Magn. Mater. 123 (1993) 107.

[43] Hong, Chin-Yih Rex, Horng,Herng-Er, U.S. Pat. No. 5954991.

[44] Chin-Yih Hong, Herng-Er Horng, F.C. Kuo, S.Y. Yang, H.C. Yang, J.M. Wu, Appl. Phys. Lett. 75 (1999) 2196.

[45] Herng-Er Horng, Chin-Yih Hong, S.L. Lee, C.H. Ho, S.Y. Yang, H.C. Yang, J. Appl. Phys. 88 (2000) 5904.

[46] J. Cernak, J. Magn. Magn. Mater. 132 (1994) 258.

[47] B. Jeyadevan, I. Nakatani, J. Magn. Magn. Mater. 201 (1999) 62.

[48] A.O. Tsebers(Cebers), Magn. Gidro., 18, 42 (1982) (in Russian). English translation: Magnetohydrodynamics 18 (1983) 345 .

[49] K. Sano, M. Doi, J. Phys. Soc. Jpn. 52 (1983) 2810.

[50] H.C. Yang, I.J. Jang, H.E. Horng, J.M. Wu, Y.C. Chiou, ChinYih Hong, J. Magn. Magn. Mater. 201 (1999) 313.
[51] Chin-Yih Hong, J. Appl. Phys. 85 (1999) 5962.

[52] F. Marty Ytreberg, Susan R. McKay, Phys. Rev. E 61 (2000) 4107.

[53] S.Y. Yang, I.J. Jang, H.E. Horng, Chin-Yih Hong, H.C. Yang, Magn. Gidro. 36 (2000) 19.

[54] Chin-Yih Hong, C.H. Ho, H.E. Horng, Chun-hui Chen, S.Y. Yang, Y.P. Chiu, H.C. Yang, Magn. Gidro. 35 (1999) 364.

[55] A.O. Tsebers, M.M. Maiorov, Sov. Tech. Phys. Lett. 6 (1980) 50.

[56] Stephen A. Langer, Raymond E. Goldstein, David P. Jackson, Phys. Rev. A 46 (1992) 4894.

[57] A.J. Dicckstein, S. Erramilli, R.E. Goldstein, D.P. Jackson, S.A. Langer, Science 261 (1993) 1012.

[58] J.-C. Bacri, A. Cebers, C. Flament, S. Lacis, R. Melliti, R. Perzynski, Progr. Colloid. Polym. Sci. 98 (1995) 30.

[59] I.J. Jang, H.E. Horng, Y.C. Chiou, Chin-Yih Hong, J.M. Wu, H.C. Yang, J. Magn. Magn. Mater. 201 (1999) 317.

[60] H.C. Yang, S.Y. Yang, W.S. Tse, Y.P. Chiu, H.E. Horng, Chin-Yih Hong, Magn. Gidro. 35 (1999) 328.

[61] E. Frederic, C. Houssier, Electric Dichroism and Electric Birefringence, Oxford University Press, Oxford, 1973.

[62] Q. Majorana, Z. Phys. 4 (1902) 145.

[63] A. Peterlin, H.A. Stuart, Z. Phys. 112 (1939) 129.

[64] M. Xu, P.J. Ridler, J. Appl. Phys. 82 (1997) 326.

[65] I. Abu-Aljarayesh, M.A. Abdallah, D. Manasrah, H. Abu-Safia, N.A. Yusuf, J. Magn. Magn. Mater. 138 (1994) 189.

[66] H.E. Horng, S.Y. Yang, S.L. Lee, J.M. Wu, J.T. Jeng, ChinYih Hong, H.C. Yang, Magn. Gidro. 36 (2000) 39.

[67] V. Socoliuc, M. Rasa, V. Sofonea, D. Bica, L. Osvath, D. Luca, J. Magn. Magn. Mater. 191 (1999) 241.

[68] James E. Martin, Kimberly M. Hill, Chris P. Tigges, Phys. Rev. E 59 (1999) 5676.

[69] H.E. Horng, in preparation.

[70] Chin-Yih Hong, J. Magn. Magn. Mater. 201 (1999) 178.

[71] H.E. Horng, private communication.

[72] H.E. Horng, S.Y. Yang, Chun-Hui Chen, Y.P. Chiu, J.A. Chen, Chin-Yih Hong, H.C. Yang, J. Magn. Mgan. Mater. 226-230 (2001) 1924.

[73] Chin-Yih Hong, Chih-Hao Lin, Chun-Hui Chen, Y.P. Chiu, S.Y. Yang, H.E. Horng, H.C. Yang, J. Magn. Magn. Mater. 226-230 (2001) 1921. 